\title{
Addressing In-Situ Challenges Using Integrated Hardware and Software
}

B.K. Miller and S. Mick

Gatan Inc. 5794 W. Las Positas Blvd. Pleasanton, CA 94588, USA

The prevalence of in-situ TEM has increased dramatically in the past 5 years. This is an indication of progress toward making in-situ experiments more accessible to researchers as well as an increased awareness of the value and importance of performing in-situ investigations.

However, there remain significant challenges to acquiring high quality data and gleaning meaningful knowledge from this data. Some of the challenges include, capturing dynamic events, reducing beam damage and sample drift, aggregating and organizing in-situ data, accurately reproducing ex-situ conditions, accurately measuring in-situ conditions, determining the effect of the electron beam, and observing a representative sample. Specific issues underlying these challenges range from straightforward technical difficulties, to pervasive problems, for which comprehensive solutions are not currently known. Nevertheless, some issues within several of the in-situ challenges indicated above have been jointly addressed by Gatan and DENSsolutions recently.

The high frame rate, large field of view, and lookback capability of the Gatan OneView camera are enabling researchers to capture dynamic events. This is demonstrated by a video sequence, depicted in Figure 1, with a field of view of over $27000 \mathrm{~nm}^{2}$ in which lattice fringes of just $0.2 \mathrm{~nm}$ are clearly resolved. In this video, a particle transforms over the course of 10.9 s, with 272 frames recording this transformation. This large field of view increases the probability that a structural change of interest will be caught, not missed because it is occurring just off-screen, and the frame rate makes it more likely that previously unobserved intermediate states will be captured.

In addition, the excellent stability of the DENSsolutions MEMS-based Wildfire heating holder significantly reduces undesired sample drift, as demonstrated by a video acquired at $1100{ }^{\circ} \mathrm{C}$ in which the sample moves just $1.6 \mathrm{~nm}$ during the 39 seconds recorded. Given the $25 \mathrm{fps}$ acquisition rate, this is just $1.6 \mathrm{pm}$ per frame on average. This will make recording quality high-resolution images at high temperatures more routine.

Recent Digital Micrograph integration of the DENSsolutions Wildfire holder with image data from the Gatan OneView camera has simplified aggregation and organization of this data significantly. This can be seenin Figure 2, which contains two screenshots of the playback of an in-situ dataset in which a reversible phase transformation was observed while oscillating the temperature using the most recent Gatan Microscopy Suite (GMS) software. The chart below the data is interactive, allowing a researcher to click on a point of known temperature and immediately see the image acquired under that precise condition. This will enable researchers to move more quickly from acquired data to scientific analysis, bypassing error-prone separate recording of temperature and tedious correlation of images with changing in-situ conditions. While other challenges remain, these successes demonstrate real progress toward making in-situ techniques more accessible. 


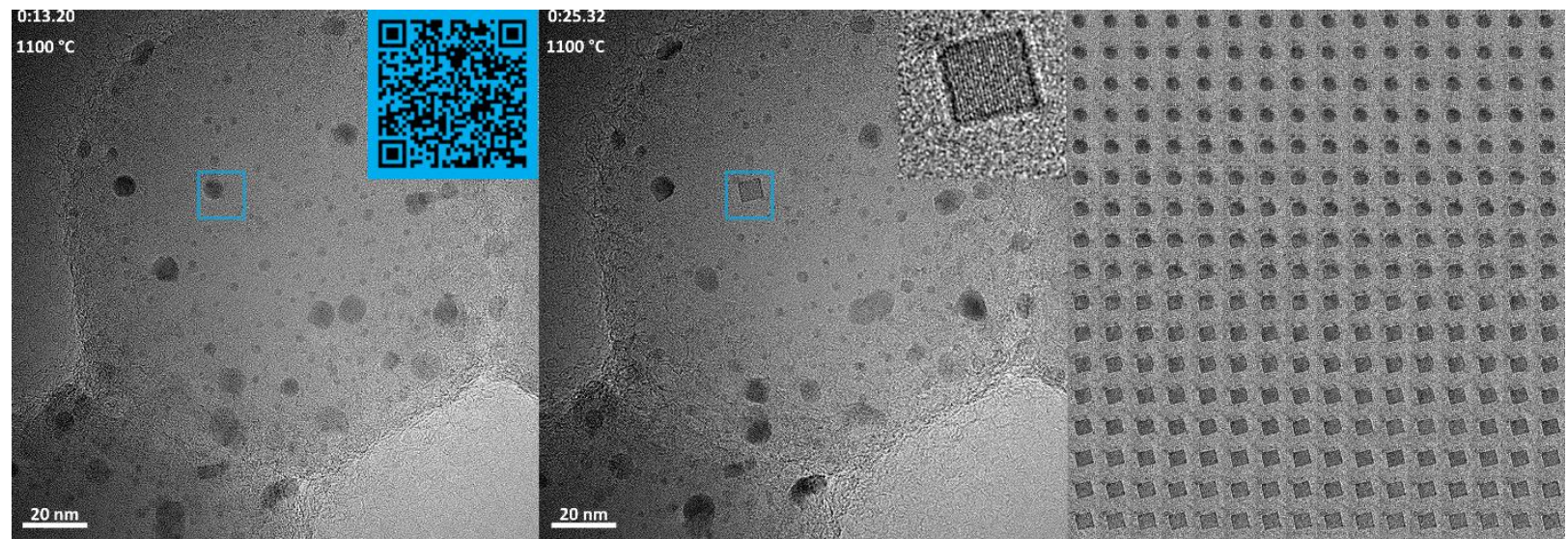

Figure 1. A silica sphere with $\mathrm{RuO}_{2}$ nanoparticles is heated to $1100{ }^{\circ} \mathrm{C}$ using a DENSsolutions Wildfire holder. One particle changes from a rounded, indistinct shape at the left to a strongly faceted shape in the center image. This shape change can be studied in detail, due to the $4 \mathrm{k} \times 4 \mathrm{k}$ OneView camera, enabling fringes to be observed in the particle, as well as the $25 \mathrm{fps}$ acquisition, which yields 272 frames from this transformation, as shown at the right. (Online video can be accessed by scanning the blue QR codes in this and the next figure.)

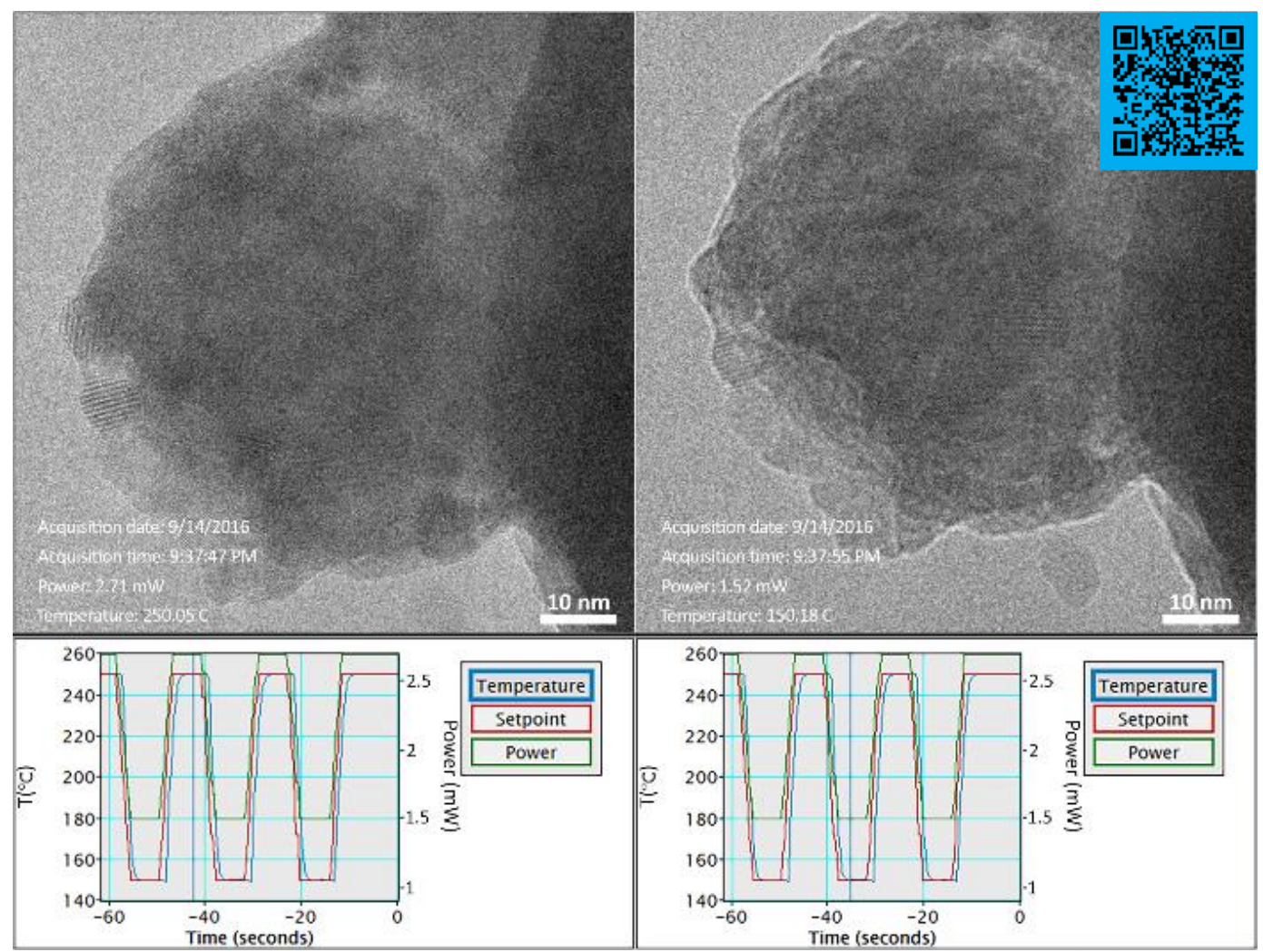

Figure 2. Data integration interactive chart. A CuSn 1:9 alloy nanoparticle (US Research Nanomaterials Inc.) is heated to temperatures just below and just above a phase transition temperature. The individual image frames from a video sequence acquired with the OneView camera are each tagged with the temperature and heater power of the DENSsolutions Wildfire holder. Clicking within the plot below the image displays the corresponding image frame from the in-situ video sequence, and playing the video updates the plot. 